\title{
The Debye'S Potentials Utilization in the Three-Dimensional Problems of the Radiation and Propagation of the Elastic Waves
}

\author{
S. L. Ilme nkov, A. A. Kleshchev* \\ Saint-Petersburg State Navy Technical University, Saint-Petersburg, Lotsmanskaya st.,3, 190008, Russia
}

\begin{abstract}
Are studied internal and external tasks of radiation of a sound by the elastic bodies, exciting by the harmonic point source, imitating turbulent pulsation of a flow of a liquid. The angular characteristics of radiation of a hollow spheroidal shell are calcu lated. The characteristic equations of the axial three-d imensional flexural waves in the hollow cylindrical shell and cylindrical bar are received with the help of Debye's potentials. The phase velocities of the various forms of these waves for shells and for cylindrical bar are calculated.
\end{abstract}

Keywords Turbulence, Flexural Wave, Longitudinal Wave, Radiation of the Sound, Phase Velocity

\section{Introduction}

At study of the three-dimensional characteristics of radiation of a sound by the elastic shells and bars exciting by the turbulent pulsation of a flow of a liquid and calculation of the phase velocities of the axial flexural waves in such bodies at such excitation it is necessary to use an artificial way of division variable in Helmholt $\mathrm{z}$ vector equation for a vector function $\vec{A}$ - to present her through Debye's potentials or "such as Debye's" potentials.

\section{The Radiation of a Sound by the Cylindrical and Spheroidal Shells, Exciting by the Turbulent Pulsation of a Flow of a Liquid}

Firstly we will consider a physical model of radiation of a sound by a cylindrical pipe (an internal task) and spheroidal shell (an external task), which is raised by turbulent pulsations of a liquid flow.

In a monograph[1], devoted to the studying of the hydrodynamic sources of a sound, it is noted that for the range of problems regarding the radiations of a sound effected by turbulent pulsations of a liquid flow, the calculation, based on the concentrated force, caused by this pulsation, gives rise to certain interest.. The similar physical model is used in a present article. Such approach is based on

* Corresponding author:

alexalex-2@yandex.ru (A. A. Kleshchev)

Published online at http://journal.sapub.org/ijtmp

Copyright (C) 2012 Scientific \& Academic Publishing. All Rights Reserved the earlier obtained results in the course of authors' research of the three-dimensional problems of diffraction and radiation of a sound by the elastic bodies of cylindrical and spheroidal forms [2 - 5].

Let's turn to an internal task for the consideration of: the harmonic point source $Q\left(b, \varphi_{1}, z_{1}\right)$ of frequency $\omega$, that imitate turbulent pulsation, is found on the interior of the shell $\left(r_{1}=b\right)$, but the point of observation $P$ with the cylindrical coordinates $r_{0}, \varphi_{0}, z_{0}$ is situated inside of a liquid flow $\left(r_{0}<b\right)$ (figure 1$)$ :

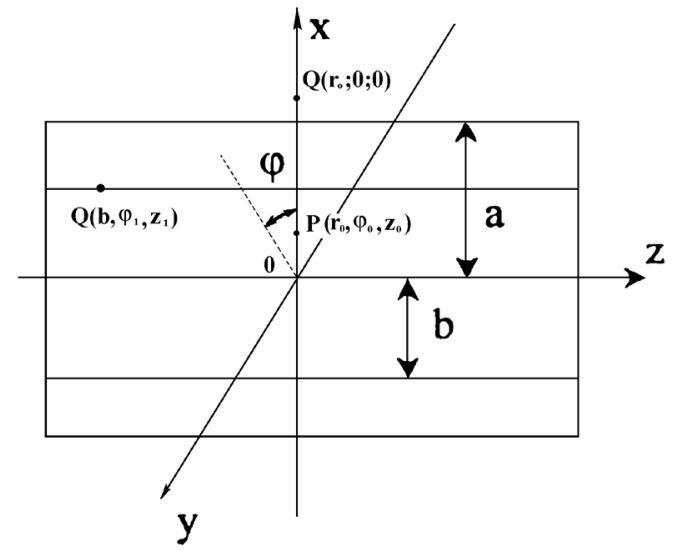

Figure 1. The isotropic cylindrical shell, exciting from within by a point harmonic source

The external radius of the cylindrical pipe $r_{0}=a$, on the outside region to the shell $\left(r_{2}=a\right)$ is the vacuum. In order to except the presence of the source (a peculiarity) on the boundary surface, we use a reciprocity theorem (6) and trade 
places a source $Q$ and a point of observation $P$. As a result the formulation of the task will be the following: we will search potential $\Phi_{s}\left(b, \varphi_{1}, z_{1}\right)$ in point $\mathrm{Q}$ of an internal border, resulting of the dispersion by pipe of a sound fro $m$ dot source with potential $\Phi_{i}\left(r_{0}, \varphi_{0}, z_{0}\right)$ placed in a point $\mathrm{P}$, basing on $[5,7]$. The potential $\Phi_{i}$ of the harmonic point source in a point $Q$ is defined by the series $[8-10]$ :

$$
\begin{aligned}
& \Phi_{i}\left(b, \phi_{1}, z_{1}\right)= \\
& \frac{i}{2} \sum_{n=0}^{\infty} \cos \left(n \phi_{1}\right) \cdot \varepsilon_{n} \int_{-\infty}^{+\infty} \exp \left(i \gamma z_{1}\right) \cdot H_{n}^{(1)}\left(k_{\gamma} b\right) \cdot J_{n}\left(k_{\gamma} r_{0}\right) d \gamma, \\
& \quad b>r_{0},
\end{aligned}
$$

where: $k_{\gamma}=\left(k^{2}-\gamma^{2}\right)^{1 / 2} ; k=\omega / c_{0} ; c_{0}$ - is the sound velocity; $\gamma$ and $k_{\gamma}$-are the axial and radial components of a wave vector $\vec{k}$ correspondingly; $\varphi_{0}=0 ; z_{0}=0$ (figure 1);

$$
\varepsilon_{n}= \begin{cases}1, & n=0 \\ 2, & n \neq 0 .\end{cases}
$$

By analogy with $\Phi_{i}$ in a point $\mathrm{Q}$, a potential of the diffused wave shell $\Phi_{s}$ can be written as[7, 5, 11]:

$$
\begin{aligned}
& \Phi_{s}\left(b, \phi_{1}, z_{1}\right)= \\
& \sum_{n=0}^{\infty} a_{n} \cos \left(n \phi_{1}\right) \cdot \int_{-\infty}^{+\infty} A(\gamma) \exp \left(i \gamma z_{1}\right) \cdot J_{n}^{(1)}\left(k_{\gamma} b\right) d \gamma,
\end{aligned}
$$

where $a_{n}$ and $A(\gamma)$ - are unknown coefficients and functions of $\gamma$ correspondingly and they are determined by the boundary conditions.

The cylindrical components of a displacement vector $\vec{U}\left(U_{r}, U_{\varphi}, U_{z}\right)$ can be written by way of potential $\Phi$ and the cylindrical components of a function $\vec{A}\left(A_{r}, A_{\varphi}, A_{z}\right)$ [12-14]:

$$
\left.\begin{array}{rl}
U_{r} & =\frac{\partial \Phi}{\partial r}+\frac{1}{r} \frac{\partial A_{z}}{\partial \varphi}-\frac{\partial A_{\varphi}}{\partial z} ; \\
U_{\varphi} & =\frac{1}{r} \frac{\partial \Phi}{\partial \varphi}+\frac{\partial A_{r}}{\partial z}-\frac{\partial A_{z}}{\partial r} ; \\
U_{z} & =\frac{\partial \Phi}{\partial z}+\frac{1}{r} A_{\varphi}+\frac{\partial A_{\varphi}}{\partial r}-\frac{1}{r} \frac{\partial A_{r}}{\partial \varphi} .
\end{array}\right\}
$$

The cylindrical components $A_{r}, A_{\varphi}, A_{z}$ of the function $\vec{A}$ can be described by way of Debye's potentials $U$ and $V[5$, 12 - 16]:

$$
\begin{aligned}
& A_{r}=\kappa^{2} r U+r \frac{\partial^{2} U}{\partial r^{2}}+z \frac{\partial^{2} U}{\partial r \partial z}+2 \frac{\partial U}{\partial r}+i \frac{1}{r} \kappa z \frac{\partial V}{\partial \varphi} \\
& A_{\varphi}=-i \kappa\left[z \frac{\partial V}{\partial r}-r \frac{\partial V}{\partial z}\right]+\frac{1}{r} \frac{\partial U}{\partial \varphi}+\frac{\partial^{2} U}{\partial r \partial \varphi}+\frac{1}{r} z \frac{\partial^{2} U}{\partial z \partial \varphi} \\
& A_{z}=\kappa^{2} z U+z \frac{\partial^{2} U}{\partial z^{2}}-i \kappa^{2} \frac{\partial V}{\partial \varphi}+r \frac{\partial^{2} U}{\partial r \partial z}+2 \frac{\partial U}{\partial z}
\end{aligned}
$$

where: $\kappa$-the wave number of the lateral wave in the shell's material;

A vector function $\vec{A}$ is are described by Debye's potentials $U$ and $V[12-16]$ :

$$
\vec{A}=\operatorname{rot} \operatorname{rot}(\vec{R} U)+i \kappa \operatorname{rot}(\vec{R} V),
$$

where: $\vec{R}$ - is a radius-vector of view point.

The efficiency of such representation becomes obvious if we take into account, that the functions $\mathrm{U}$ and $\mathrm{V}$ submit to the Helmholtz scalar equation, divided in circular cy lindrical coordinates:

$$
\begin{aligned}
& \left(\Delta+\kappa^{2}\right) U=0 \\
& \left(\Delta+\kappa^{2}\right) V=0
\end{aligned}
$$

The other representations for a vector function $\vec{A}$ in the Cartesian and cylindrical coordinate systems are given in[17 - 19], but it is [5] in a spheroidal system.

The potentials $\Phi, U$ and $V$ are also expanded in serieses by eigen-functions of the Helmholtz scalar equation[16, 5, 11]:

$$
\begin{aligned}
& \Phi(r, \phi, z)=\sum_{n=0}^{\infty} \cos (n \phi)\left[b_{n} \int_{-\infty}^{+\infty} B(\gamma) \exp (i \gamma z) J_{n}\left(h_{\gamma} r\right) d \gamma+b_{n}^{\prime} \int_{-\infty}^{+\infty} B^{\prime}(\gamma) \exp (i \gamma z) N_{n}\left(h_{\gamma} r\right) d \gamma\right] \\
& U(r, \phi, z)=\sum_{n=1}^{\infty} \sin (n \phi)\left[c_{n} \int_{-\infty}^{+\infty} C(\gamma) \exp (i \gamma z) J_{n}\left(\kappa_{\gamma} r\right) d \gamma+c_{n}^{\prime} \int_{-\infty}^{+\infty} C^{\prime}(\gamma) \exp (i \gamma z) N_{n}\left(\kappa_{\gamma} r\right) d \gamma\right] \\
& V(r, \phi, z)=\sum_{n=0}^{\infty} \cos (n \phi)\left[d_{n} \int_{-\infty}^{+\infty} D(\gamma) \exp (i \gamma z) J_{n}\left(\kappa_{\gamma} r\right) d \gamma+d_{n}^{\prime} \int_{-\infty}^{+\infty} D^{\prime}(\gamma) \exp (i \gamma z) N_{n}\left(\kappa_{\gamma} r\right) d \gamma\right]
\end{aligned}
$$

где: $h_{\gamma}=\left(k_{l}^{2}-\gamma^{2}\right)^{1 / 2} ; k_{l}=\omega / c_{1}$ - is a longitudinal wave number; $c_{1}$ - is a longitudinal wave velocity in the shell's material; $N_{n}\left(\kappa_{\gamma} r\right)$ - Neiman's cylindrical; $\kappa_{\gamma}=\left(\kappa^{2}-\gamma^{2}\right)^{1 / 2} ; b_{n}, b_{n}^{\prime}, c_{n}, c_{n}^{\prime}, d_{n}, d_{n}^{\prime}$ и $B(\gamma), B^{\prime}(\gamma)$, $C(\gamma), C^{\prime}(\gamma), D(\gamma), D^{\prime}(\gamma)$ - are unknown coefficients and functions correspondingly and they are determined by the next boundary conditions at the external and internal surfaces of an elastic shell: 
1) a normal component of a displacement vector $U_{r}$ is continuous at an internal shell's boundary;

2) a sound pressure in a fluid is equal to the normal strain in a shell at an internal boundary;

3) a normal strain in a shell at an external boundary is equal to zero;

4) the tangent strains at the shell's boundaries are equal to zero.

An analytic form of the enumerated boundary conditions are a following representation:

$$
\begin{aligned}
& \frac{\partial \Phi}{\partial r}+\frac{1}{r} \frac{\partial A_{z}}{\partial \phi}-\frac{\partial A_{\phi}}{\partial z}=\left.\frac{\partial}{\partial r}\left(\Phi_{i}+\Phi_{s}\right)\right|_{r=b} \\
& (\lambda+2 \mu) \frac{\partial U_{r}}{\partial r}+\lambda\left(\frac{1}{r} \frac{\partial U_{\phi}}{\partial \phi}+\frac{1}{r} U_{r}+\frac{\partial U_{z}}{\partial z}\right)=-\left.\rho_{0} \omega^{2}\left(\Phi_{i}+\Phi_{s}\right)\right|_{r=b} \\
& (\lambda+2 \mu) \frac{\partial U_{r}}{\partial r}+\lambda\left(\frac{1}{r} \frac{\partial U_{\phi}}{\partial \phi}+\frac{1}{r} U_{r}+\frac{\partial U_{z}}{\partial z}\right)=\left.0\right|_{r=a} \\
& \frac{\partial U_{\phi}}{\partial r}-\frac{1}{r} U_{\phi}+\frac{1}{r} \frac{\partial U_{r}}{\partial \phi}=\left.0\right|_{\substack{r=a \\
r=b}} \\
& \frac{\partial U_{r}}{\partial z}+\frac{\partial U_{z}}{\partial r}=\left.0\right|_{\substack{r=a \\
r=b}}
\end{aligned}
$$

A substitution of the series (1), (2), (10) - (12) in the boundary conditions (13) - (17) results in an infinite system of the equations to define the unknown coefficients and functions $a_{n} A(\gamma) ;, b_{n} B(\gamma), b_{n}^{\prime} B^{\prime}(\gamma), c_{n} C(\gamma), c_{n}^{\prime} C^{\prime}(\gamma)$, $d_{n} D(\gamma), d_{n}^{\prime} D^{\prime}(\gamma)$. As the trigonometrical functions $\cos (n \varphi)$ and $\sin (n \varphi)$ are opthogonal, an infinite system breaks out into seven equation with fixed index $\mathrm{n}$ for finding the seven combinations of the unknown coefficients and functions.

A product $a_{n} A(\gamma)$ for a potential of a diffused wave $\Phi_{s}$ is calculated by the Cramer rule on a basis of a ratio of the who determinants of the seven degree:

$$
a_{n} A(\gamma)=\Delta^{\prime} / \Delta
$$

where: $\Delta-$ is determinant of a system, but $\Delta^{\prime}$-is minor;

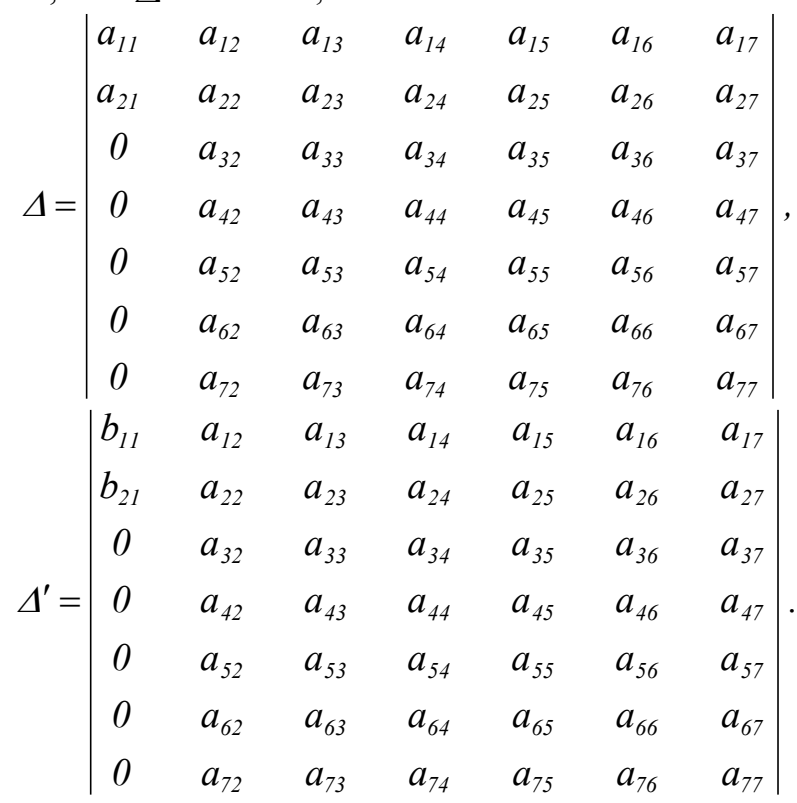

$$
\begin{aligned}
& a_{11}=-J_{n}^{\prime}\left(k_{\gamma} b\right) \text {; } \\
& a_{21}=-\rho_{0} \omega^{2} J_{n}\left(k_{\gamma} b\right) \text {; } \\
& b_{11}=-\frac{i}{2} \varepsilon_{n} H_{n}^{(1)}{ }^{\prime}\left(k_{\gamma} b\right) J_{n}\left(k_{\gamma} r_{0}\right) \text {; } \\
& b_{21}=-\rho_{0} \omega^{2} \frac{i}{2} \varepsilon_{n} H_{n}^{(I)}\left(k_{\gamma} b\right) J_{n}\left(k_{\gamma} r_{0}\right)
\end{aligned}
$$


The rest of elements of the rows and columnes of a determinants $\Delta$ и $\Delta^{\prime}$ can be taken out[5]. Except for the first column elements $\Delta$ and $\Delta^{\prime}$ - identical.

An influence of a turbulent pulsation at a prolate spheroidal shell is considered as an external problem. At figure 2 is show hollow spheroidal shell, by streamline flow of a liquid. The points $A, B, C$ mark the possible positions of a point source, imitating turbulent pulsation. With help [2 - 4], can be calculated the angular characteristics of a radiation of a spheroidal shell under an influence of a turbulent pulsation.

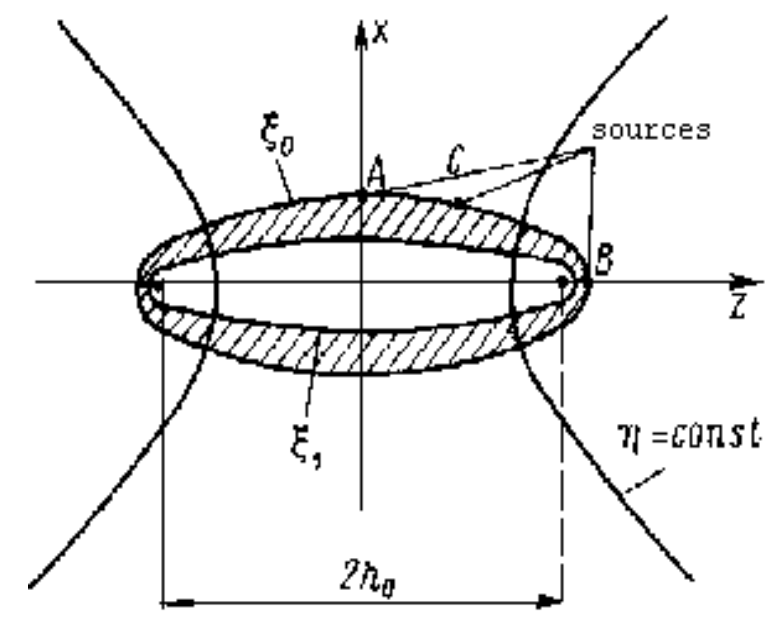

Figure 2. The isotropic prolate spheroidal shell, exciting on the out side by a point harmonic source

At figures 3 and 4 are shown the modulus of the angular characteristics of a radiation $\left|F_{l}(\theta ; \varphi)\right|$ this shell by an excitement in a point $B$ (figure 3 ) and point $A$ (figure 4). $c=k h_{0}$ is a wave dimension of a body, $h_{0}$ - is a half of an interfocus distance (figure 2). A figure 3 corresponds $C=8,1$, but a figure $4-C=5,5$.

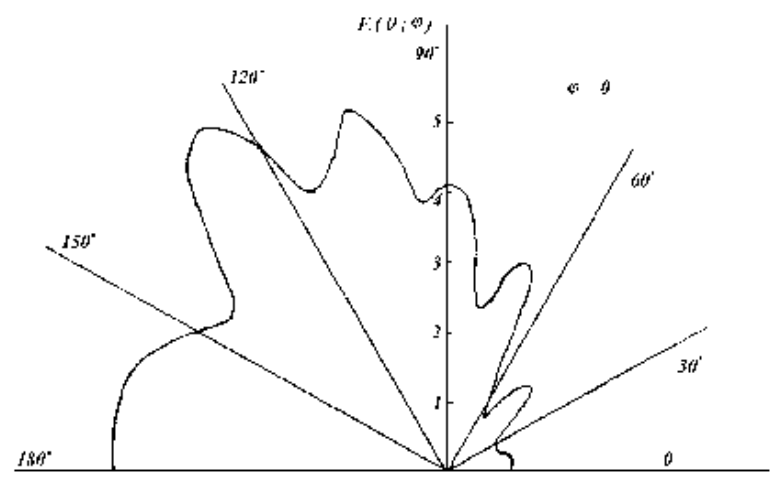

Figure 3. The modulus of an angular characteristic of a radiation $\left|F_{l}(\theta ; \varphi)\right|$ hollow spheroidal shell, exciting on the exciting on the outside by a point harmonic source in a point $B ; C=8,1$

A dipole character of a radiation of a turbulent pulsation[1] can foresee an introduction of a second source (with an other sign), disposed at a small from a first source, and to calculate in a point of observation total pressure from two sources. By an use of a reciprocity theorem a transference of a turbulent pulsation $Q$ together with a flow of a fluid along of an internal surface of a shell (figure 1) is substituted for a transference of a point of observation $P$ parallel to a boundary in a opposite direction.

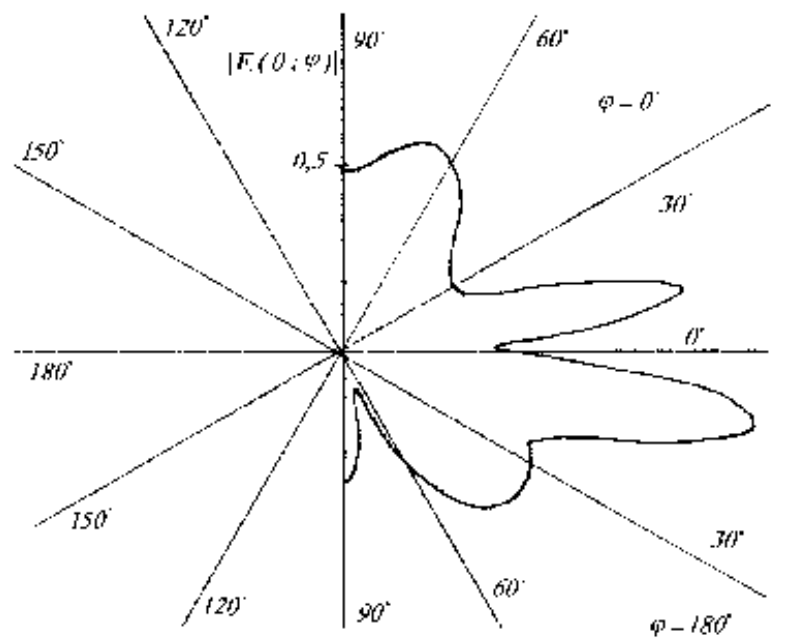

Figure 4. The modulus of an angular characteristic of a radiation $\left|F_{1}(\theta ; \varphi)\right|$ of a steel of a steel hollow spheroidal shell, out side by a point harmonic source in a point $A ; C=5,5$

\section{The Phase Velocities of Three-Dimensional Flexural Waves in Cylindrical Shells and Bars}

Further we shall proceed to consideration of phase velocities of one of types of waves existing in cylindrical shells and bars: axial three-dimensional flexural waves. Wave of a similar type were in detail investigated in $[5,15$, 20 - 28]. The characteristic equation for wave numbers of three-dimensional flexural waves of form $\mathrm{m}$ in the isotropic cylindrical shell it turns out by equating to zero of the determinant $\Delta$ of six order $[5,15,23,25,27,28]$ :

$\begin{array}{llllll}a_{11} & a_{12} & a_{13} & a_{14} & a_{15} & a_{16} \\ a_{21} & a_{22} & a_{23} & a_{24} & a_{25} & a_{26} \\ a_{31} & a_{32} & a_{33} & a_{34} & a_{35} & a_{36}\end{array}$

$\Delta=$

$$
\begin{array}{lllllll}
\mathrm{a}_{41} & \mathrm{a}_{42} & \multicolumn{2}{c}{\mathrm{a}_{43}} & \mathrm{a}_{44} & \mathrm{a}_{45} & \mathrm{a}_{46}=0 ; \\
\mathrm{a}_{51} & \mathrm{a}_{52} & \mathrm{a}_{53} & \mathrm{a}_{54} & \mathrm{a}_{55} & \mathrm{a}_{56} \\
\mathrm{a}_{61} & \mathrm{a}_{62} & \mathrm{a}_{63} & \mathrm{a}_{64} & \mathrm{a}_{65} & \mathrm{a}_{66}
\end{array}
$$

where : $a_{11}=-(\lambda+2 \mu) J_{m}{ }^{11}\left(h^{1} a\right)+\lambda\left[a^{-2} m^{2} J_{m}\left(h{ }^{1} a\right)-\right.$ $\left.\mathrm{a}^{-1} J_{\mathrm{m}}{ }^{1}\left(\mathrm{~h}^{1} \mathrm{a}\right)+\mathrm{k}^{2} \mathrm{~J}_{\mathrm{m}}\left(\mathrm{h}^{1} \mathrm{a}\right)\right]$;

$a_{12}=-(\lambda+2 \mu) N_{m}{ }^{11}\left(h^{1} a\right)+\lambda\left[a^{-2} m^{2} N_{m}\left(h^{1} a\right)-a^{-1} N_{m}{ }^{1}\left(h^{1} a\right)+\right.$ $\left.\mathrm{k}^{2} \mathrm{~N}_{\mathrm{m}}\left(\mathrm{h}^{1} \mathrm{a}\right)\right]$

$\mathrm{a}_{13}=(\lambda+2 \mu)\left\{-\mathrm{i} \chi \mathrm{m}^{2}\left[\mathrm{~J}_{\mathrm{m}}\left(\chi^{1} \mathrm{a}\right)-\mathrm{J}_{\mathrm{m}}{ }^{1}\left(\chi^{1} \mathrm{a}\right)\right]+\mathrm{i} \chi \mathrm{J}_{\mathrm{m}}{ }^{11}\left(\chi^{1} \mathrm{a}\right)+\right.$ $\left.i \chi k^{2}\left[J_{m}\left(\chi^{1} a\right)+a J_{m}{ }^{1}\left(\chi^{1} a\right)\right]\right\}+\lambda\left\{i a^{-2} J_{m}\left(\chi^{1} a\right)\left(m^{2}+k^{2}\right)-i \chi a^{-1}\right.$ $\left.\mathrm{J}_{\mathrm{m}}{ }^{1}\left(\chi^{1} \mathrm{a}\right)\left(\mathrm{m}^{2}+\mathrm{k}^{2}\right)-2 \mathrm{i} \chi \mathrm{k}^{2} \mathrm{~J}_{\mathrm{m}}\left(\chi^{1} \mathrm{a}\right)-\mathrm{i} \chi \mathrm{J}_{\mathrm{m}}{ }^{11}\left(\chi^{1} \mathrm{a}\right)\right\}$;

$\mathrm{a}_{14}=(\lambda+2 \mu)\left\{-1 \chi \mathrm{m}^{2}\left[\mathrm{~N}_{\mathrm{m}}\left(\chi^{1} \mathrm{a}\right)-\mathrm{N}_{\mathrm{m}}{ }^{1}\left(\chi^{1} \mathrm{a}\right)\right]+\mathrm{i} \chi \mathrm{N}_{\mathrm{m}}{ }^{11}\left(\chi^{1} \mathrm{a}\right)+\right.$ $\left.\mathrm{i}_{\chi} \mathrm{k}^{2}\left[\mathrm{~N}_{\mathrm{m}}\left(\chi^{1} \mathrm{a}\right)+\mathrm{a} \mathrm{N}_{\mathrm{m}}^{1}\left(\chi^{1} \mathrm{a}\right)\right]\right\}+\lambda\left\{\mathrm{i} \chi \mathrm{a}^{-2} \mathrm{~N}_{\mathrm{m}}\left(\chi^{1} \mathrm{a}\right)\left(\mathrm{m}^{2}+\mathrm{k}^{2}\right)-\right.$ i $\left.\chi \mathrm{a}^{-1} \mathrm{~N}_{\mathrm{m}}{ }^{1}\left(\chi^{1} \mathrm{a}\right)\left(\mathrm{m}^{2}+\mathrm{k}^{2}\right)--2 i \chi \mathrm{k}^{2} \mathrm{~N}_{\mathrm{m}}\left(\chi^{1} \mathrm{a}\right)-\mathrm{i} \chi \mathrm{N}_{\mathrm{m}}{ }^{11}\left(\chi^{1} \mathrm{a}\right)\right\}$; $\mathrm{a}_{15}=2(\lambda+2 \mu)\left(\mathrm{a}^{-2}-1\right) \mathrm{ikm} \mathrm{J}_{\mathrm{m}}\left(\chi^{1} \mathrm{a}\right) ; \mathrm{a}_{16}=2(\lambda+$ $2 \mu)\left(a^{-2}-1\right) i k m N_{m}\left(\chi^{1} a\right)$

$a_{21}=-(\lambda+2 \mu) J_{m}^{11}\left(h^{1} b\right)+\lambda\left[b^{-2} m^{2} J_{m}\left(h^{1} b\right)-b^{-1} J_{m}{ }^{1}\left(h^{1} b\right)+k^{2}\right.$ $\left.\mathrm{J}_{\mathrm{m}}\left(\mathrm{h}^{\mathrm{l}} \mathrm{b}\right)\right]$; 
$a_{22}=-(\lambda+2 \mu) N_{m}{ }^{11}\left(h^{1} b\right)+\lambda\left[b^{-2} m^{2} N_{m}\left(h^{1} b\right)-b^{-1} N_{m}{ }^{1}\left(h^{1} b\right)+\right.$ $\left.\mathrm{k}^{2} \mathrm{~N}_{\mathrm{m}}\left(\mathrm{h}^{1} \mathrm{~b}\right)\right]$

$\mathrm{a}_{23}=(\lambda+2 \mu)\left\{-\mathrm{i} \chi \mathrm{m}^{2}\left[\mathrm{~J}_{\mathrm{m}}\left(\chi^{1} \mathrm{~b}\right)-\mathrm{J}_{\mathrm{m}}{ }^{1}\left(\chi^{1} \mathrm{~b}\right)\right]+\mathrm{i} \chi \mathrm{J}_{\mathrm{m}}{ }^{11}\left(\chi^{1} \mathrm{~b}\right)+\right.$ $\left.\mathrm{i} \chi \mathrm{k}^{2}\left[\mathrm{~J}_{\mathrm{m}}\left(\chi^{1} \mathrm{~b}\right)+\mathrm{b} \mathrm{J}_{\mathrm{m}}^{1}\left(\chi^{1} \mathrm{~b}\right)\right]\right\}+\lambda\left\{\mathrm{i} \chi \mathrm{b}^{-2} \mathrm{~J}_{\mathrm{m}}\left(\chi^{1} \mathrm{~b}\right)\left(\mathrm{m}^{2}+\mathrm{k}^{2}\right)-\right.$ $\left.\mathrm{i} \chi \mathrm{b}^{-1} \mathrm{~J}_{\mathrm{m}}{ }^{1}\left(\chi^{1} \mathrm{~b}\right)\left(\mathrm{m}^{2}+\mathrm{k}^{2}\right)-2 \mathrm{i} \chi \mathrm{k}^{2} \mathrm{~J}_{\mathrm{m}}\left(\chi^{1} \mathrm{~b}\right)-\mathrm{i} \chi \mathrm{J}_{\mathrm{m}}{ }^{11}\left(\chi^{1} \mathrm{~b}\right)\right\}$;

$\mathrm{a}_{24}=(\lambda+2 \mu)\left\{-\mathrm{i} \chi \mathrm{m}^{2}\left[\mathrm{~N}_{\mathrm{m}}\left(\chi^{1} \mathrm{~b}\right)-\mathrm{N}_{\mathrm{m}}{ }^{1}\left(\chi^{1} \mathrm{~b}\right)\right]+\mathrm{i} \chi \mathrm{N}_{\mathrm{m}}{ }^{11}\left(\chi^{1} \mathrm{~b}\right)\right.$ $\left.+\mathrm{i} \chi \mathrm{k}^{2}\left[\mathrm{~N}_{\mathrm{m}}\left(\chi^{1} \mathrm{~b}\right)+\mathrm{b} \mathrm{N} \mathrm{N}^{1}\left(\chi^{1} \mathrm{~b}\right)\right]\right\}+\lambda\left\{\mathrm{i} \chi \mathrm{b}^{-2} \mathrm{~N}_{\mathrm{m}}\left(\chi^{1} \mathrm{~b}\right)\left(\mathrm{m}^{2}+\mathrm{k}^{2}\right)-\right.$ $\left.\mathrm{i} \chi \mathrm{b}^{-1} \mathrm{~N}_{\mathrm{m}}^{1}\left(\chi^{1} \mathrm{~b}\right)\left(\mathrm{m}^{2}+\mathrm{k}^{2}\right)-2 \mathrm{i} \chi \mathrm{k}^{2} \mathrm{~N}_{\mathrm{m}}\left(\chi^{1} \mathrm{~b}\right)-\mathrm{i} \chi \mathrm{N}_{\mathrm{m}}{ }^{11}\left(\chi^{1} \mathrm{~b}\right)\right\}$;

$\mathrm{a}_{25}=2(\lambda+2 \mu)\left(\mathrm{b}^{-2}-1\right) \mathrm{ikm} \mathrm{J}_{\mathrm{m}}\left(\chi^{1} \mathrm{~b}\right) ; \mathrm{a}_{26}=2(\lambda+$ $2 \mu)\left(b^{-2}-1\right)$ ikm $N_{m}\left(\chi^{1} b\right)$;

$a_{31}=2 m a^{-1}\left[J_{m}{ }^{1}\left(h^{1} a\right)-a^{-1} J_{m}\left(h^{1} a\right)\right] ; a_{32}=2 m a^{-1}\left[N_{m}{ }^{1}\left(h^{1} a\right)-\right.$ $\left.\mathrm{a}^{-1} \mathrm{~N}_{\mathrm{m}}\left(\mathrm{h}^{1} \mathrm{a}\right)\right]$

$a_{33}=i \chi m\left\{J_{m}\left(\chi^{1} a\right)\left[a^{-2}\left(2-m^{2}\right)-k^{2}\right]-J_{m}{ }^{11}\left(\chi^{1} a\right)-a^{-1}\right.$ $\left.\mathrm{J}_{\mathrm{m}}{ }^{1}\left(\chi^{1} \mathrm{a}\right)\right\}$

$\mathrm{a}_{34}=\mathrm{i} \chi \mathrm{m}\left\{\mathrm{N}_{\mathrm{m}}\left(\chi^{1} \mathrm{a}\right)\left[\mathrm{a}^{-2}\left(2-\mathrm{m}^{2}\right)-\mathrm{k}^{2}\right]-\mathrm{N}_{\mathrm{m}}{ }^{11}\left(\chi^{1} \mathrm{a}\right)-\mathrm{a}^{-1}\right.$ $\left.\mathrm{N}_{\mathrm{m}}{ }^{1}\left(\chi^{1} \mathrm{a}\right)\right\}$;

$\mathrm{a}_{35}=\mathrm{ikJ}_{\mathrm{m}}{ }^{1}\left(\chi^{1} \mathrm{a}\right) \mathrm{a} \chi^{2} ; \quad \mathrm{a}_{36}=\mathrm{ikN}{ }_{\mathrm{m}}^{1}\left(\chi^{1} \mathrm{a}\right) \mathrm{a} \chi^{2}$;

$\mathrm{a}_{41}=2 \mathrm{mb}^{-1}\left[\mathrm{~J}_{\mathrm{m}}{ }^{1}\left(\mathrm{~h}^{1} \mathrm{~b}\right)-\mathrm{b}^{-1} \mathrm{~J}_{\mathrm{m}}\left(\mathrm{h}^{1} \mathrm{~b}\right)\right] ; \mathrm{a}_{42}=2 \mathrm{mb}^{-1}\left[\mathrm{~N}_{\mathrm{m}}{ }^{1}\left(\mathrm{~h}^{1} \mathrm{~b}\right)-\right.$ $\left.\mathrm{b}^{-1} \mathrm{~N}_{\mathrm{m}}\left(\mathrm{h}^{1} \mathrm{~b}\right)\right]$;

$a_{43}=i \chi m\left\{J_{m}\left(\chi^{1} b\right)\left[b^{-2}\left(2-m^{2}\right)-k^{2}\right]-J_{m}{ }^{11}\left(\chi^{1} b\right)-b^{-1}\right.$ $\left.\mathrm{J}_{\mathrm{m}}^{1}\left(\chi^{1} \mathrm{~b}\right)\right\}$;

$\mathrm{a}_{44}=\mathrm{i} \chi \mathrm{m}\left\{\mathrm{N}_{\mathrm{m}}\left(\chi^{1} \mathrm{~b}\right)\left[\mathrm{b}^{-2}\left(2-\mathrm{m}^{2}\right)-\mathrm{k}^{2}\right]-\mathrm{N}_{\mathrm{m}}{ }^{11}\left(\chi^{1} \mathrm{~b}\right)-\mathrm{b}^{-1}\right.$ $\left.\mathrm{N}_{\mathrm{m}}{ }^{1}\left(\chi^{1} \mathrm{~b}\right)\right\}$;

$\mathrm{a}_{45}=\mathrm{ikJ}_{\mathrm{m}}^{1}\left(\chi^{1} \mathrm{~b}\right) \mathrm{b} \chi^{2} ; \mathrm{a}_{46}=\mathrm{ikN}_{\mathrm{m}}^{1}\left(\chi^{1} \mathrm{~b}\right) \mathrm{b} \chi^{2}$;

$\mathrm{a}_{51}=-2 \mathrm{ik} \mathrm{J}_{\mathrm{m}}{ }^{1}\left(\mathrm{~h}^{1} \mathrm{a}\right) ; \mathrm{a}_{52}=-2 \mathrm{ik} \mathrm{N}_{\mathrm{m}}{ }^{1}\left(\mathrm{~h}^{1} \mathrm{a}\right)$;

$\mathrm{a}_{53}=-\mathrm{k} \chi\left[\mathrm{J}_{\mathrm{m}}\left(\chi^{1} \mathrm{a}\right)\left(\mathrm{m}^{2}+\mathrm{a}^{-1} \mathrm{k}^{2}\right)+5 \mathrm{~J}_{\mathrm{m}}{ }^{1}\left(\chi^{1} \mathrm{a}\right)+\mathrm{aJ}_{\mathrm{m}}{ }^{11}\left(\chi^{1} \mathrm{a}\right)\right]$;

$\mathrm{a}_{54}=-\mathrm{k} \chi\left[\mathrm{N}_{\mathrm{m}}\left(\chi^{1} \mathrm{a}\right)\left(\mathrm{m}^{2}+\mathrm{a}^{-1} \mathrm{k}^{2}\right)+5 \mathrm{~N}_{\mathrm{m}}{ }^{1}\left(\chi^{1} \mathrm{a}\right)+\mathrm{aN}_{\mathrm{m}}{ }^{11}\left(\chi^{1} \mathrm{a}\right)\right]$;

$\mathrm{a}_{55}=\chi^{2} \mathrm{~m}\left[\mathrm{a}^{-1} \mathrm{~J}_{\mathrm{m}}\left(\chi^{1} \mathrm{a}\right)-\mathrm{J}_{\mathrm{m}}{ }^{1}\left(\chi^{1} \mathrm{a}\right)\right] ; \quad \mathrm{a}_{56}=\chi^{2} \mathrm{~m}\left[\mathrm{a}^{-1} \mathrm{~N}_{\mathrm{m}}\left(\chi^{1} \mathrm{a}\right)-\right.$ $\left.\mathrm{N}_{\mathrm{m}}^{1}\left(\chi^{1} \mathrm{a}\right)\right]$

$\mathrm{a}_{61}=-2 \mathrm{ik} \mathrm{J}_{\mathrm{m}}{ }^{1}\left(\mathrm{~h}^{1} \mathrm{~b}\right) ; \quad \mathrm{a}_{62}=-2 \mathrm{ik} \mathrm{N}{ }_{\mathrm{m}}{ }^{1}\left(\mathrm{~h}^{1} \mathrm{~b}\right)$;

$\mathrm{a}_{63}=-\mathrm{k} \chi\left[\mathrm{J}_{\mathrm{m}}\left(\chi^{1} \mathrm{~b}\right)\left(\mathrm{m}^{2}+\mathrm{b}^{-1} \mathrm{k}^{2}\right)+5 \mathrm{~J}_{\mathrm{m}}{ }^{1}\left(\chi^{1} \mathrm{~b}\right)+\mathrm{bJ}_{\mathrm{m}}{ }^{11}\left(\chi^{1} \mathrm{~b}\right)\right]$;

$\mathrm{a}_{64}=-\mathrm{k} \chi\left[\mathrm{N}_{\mathrm{m}}\left(\chi^{1} \mathrm{~b}\right)\left(\mathrm{m}^{2}+\mathrm{b}^{-1} \mathrm{k}^{2}\right)+5 \mathrm{~N}_{\mathrm{m}}^{1}\left(\chi^{1} \mathrm{~b}\right)+\right.$ $\left.\mathrm{bN}_{\mathrm{m}}{ }^{11}\left(\chi^{1} \mathrm{~b}\right)\right]$

$\mathrm{a}_{65}=\chi^{2} \mathrm{~m}\left[\mathrm{~b}^{-1} \mathrm{~J}_{\mathrm{m}}\left(\chi^{1} \mathrm{~b}\right)-\mathrm{J}_{\mathrm{m}}{ }^{1}\left(\chi^{1} \mathrm{~b}\right)\right] ; \mathrm{a}_{66}=\chi^{2} \mathrm{~m}\left[\mathrm{~b}^{-1} \mathrm{~N}_{\mathrm{m}}\left(\chi^{1} \mathrm{~b}\right)-\right.$ $\left.\mathrm{N}_{\mathrm{m}}{ }^{1}\left(\chi^{1} \mathrm{~b}\right)\right]$;

In the figures 5 and 6 the results of calculations of phase velocities of three-dimensional flexural waves for steel and aluminium shells are represented. The following designations one can find : the curves 1,2 corresponds $m=1$ (zero mode); the curves $3,4-\mathrm{m}=2$; the curves $5,6-\mathrm{m}=3$; the curves 7,8- $\mathrm{m}=4$; The external radius of shells and was accepted equal 1,0 m. $\mathrm{C}_{1}-$ the veloc ity of longitudinal wave ; $C_{R}$ - the velocity of Relay's wave; $\Lambda=\left(2 \pi c_{1} / \omega\right)$; internal radius $b=0,99 \mathrm{M}$ corresponds to a thin shell, $\mathrm{b}=0,8 \mathrm{M}-$ thick shell.

The characteristic equation for wave numbers $\kappa$ of three-dimensional flexural waves in the is otropic cylindrical bar of radius $a=1,0$, received with the help of Debye's potentials, looks like[22, 26]:

$$
\begin{gathered}
\left\{-(\lambda+2 \mu) \mathrm{J}_{\mathrm{m}}{ }^{11}\left(\mathrm{~h}^{1}\right)+\lambda\left[\left(\mathrm{m}^{2}+\mathrm{k}^{2}\right) \mathrm{J}_{\mathrm{m}}\left(\mathrm{h}^{1}\right)-\mathrm{J}_{\mathrm{m}}{ }^{1}\left(\mathrm{~h}^{1}\right)\right]\right\} \\
\left\{\mathrm{ikm}\left\{\left[2-\left(\mathrm{m}^{2}+\mathrm{k}^{2}\right)\right] \mathrm{J}_{\mathrm{m}}\left(\mathrm{k}^{1}\right)-\mathrm{J}_{\mathrm{m}}{ }^{1}\left(\mathrm{k}^{1}\right)-\mathrm{J}_{\mathrm{m}}{ }^{11}\left(\mathrm{k}^{1}\right)\right\} \times\right. \\
\left\{\mathrm{k}^{2} \mathrm{~m}\left[\mathrm{~J}_{\mathrm{m}}\left(\mathrm{k}^{1}\right)-\mathrm{J}_{\mathrm{m}}{ }^{1}\left(\mathrm{k}^{1}\right)\right]\right\}+\left\{2 \mathrm { i } \mu \mathrm { k } \left[\left(\mathrm{k}^{2}-\mathrm{m}^{2}\right) \mathrm{J}_{\mathrm{m}}\left(\mathrm{k}^{1}\right)+\right.\right. \\
\left.\left.\left(\mathrm{m}^{2}+\mathrm{k}^{2}\right) \mathrm{J}_{\mathrm{m}}{ }^{1}\left(\mathrm{k}^{1}\right)+\mathrm{J}_{\mathrm{m}}{ }^{11}\left(\mathrm{k}^{1}\right)\right]\right\}\left[\mathrm{ikk}^{2}\left[\mathrm{~J}_{\mathrm{m}}{ }^{1}\left(\mathrm{k}^{1}\right)\right] \times\right. \\
\left.\left.\left[-2 \mathrm{ikJ} \mathrm{J}_{\mathrm{m}}{ }^{1}\left(\mathrm{~h}^{1}\right)\right]-\left\{\mathrm{kk}^{1}\left(\mathrm{~m}^{2}+\mathrm{k}^{2}\right)\right] \mathrm{J}_{\mathrm{m}}\left(\mathrm{k}^{1}\right)+5 \mathrm{~J}_{\mathrm{m}}{ }^{1}\left(\mathrm{k}^{1}\right)+\mathrm{J}_{\mathrm{m}}{ }^{11}\left(\mathrm{k}^{1}\right)\right]\right\} \\
{\left[-(\lambda+2 \mu) \mathrm{J}_{\mathrm{m}}{ }^{11}\left(\mathrm{~h}^{1}\right)+\lambda\left[\left(\mathrm{m}^{2}+\mathrm{k}^{2}\right) \mathrm{J}_{\mathrm{m}}\left(\mathrm{h}^{1}\right)-\mathrm{J}_{\mathrm{m}}{ }^{1}\left(\mathrm{~h}^{1}\right)\right]\right\}-} \\
\left\{\mathrm{k}^{2} \mathrm{~m}\left[\mathrm{~J}_{\mathrm{m}}\left(\mathrm{k}^{1}\right)-\mathrm{J}_{\mathrm{m}}{ }^{1}\left(\mathrm{k}^{1}\right)\right]\right\}\left\{2 \mathrm{~m}\left[\mathrm{~J}_{\mathrm{m}}{ }^{1}\left(\mathrm{~h}^{1}\right)-\mathrm{J}_{\mathrm{m}}\left(\mathrm{h}^{1}\right)\right]\right\}
\end{gathered}
$$

$\left\{2 \mathrm{i} \mu \mathrm{k}\left[\left(\mathrm{k}^{2}-\mathrm{m}^{2}\right) \mathrm{J}_{\mathrm{m}}\left(\mathrm{k}^{1}\right)+\left(\mathrm{m}^{2}+\mathrm{k}^{2}\right) \mathrm{J}_{\mathrm{m}}{ }^{1}\left(\mathrm{k}^{1}\right)+\mathrm{J}_{\mathrm{m}}{ }^{11}\left(\mathrm{k}^{1}\right)\right]\right\}=0 .(20)$ In the figure 7 the phase velocities of first three forms of flexu ral waves in the steel cylindrical bar, received with the help of "such as Debye's" potentials, are represented.[19, 28].

The the phase velocities of axissymmetrical flexural waves (longitudinal and torsional in bars, longitudinal, torsional and flexural in shells) are submitted in [5, 24, 26, $27,28]$. As to anisotropic of shells and bars, the jobs are devoted to phase velocities of elastic waves in them[18, 29, 30].

On known phase velocities the components of a vector of displacement of an elastic body in any point with the help of results of job can be calculated[31].

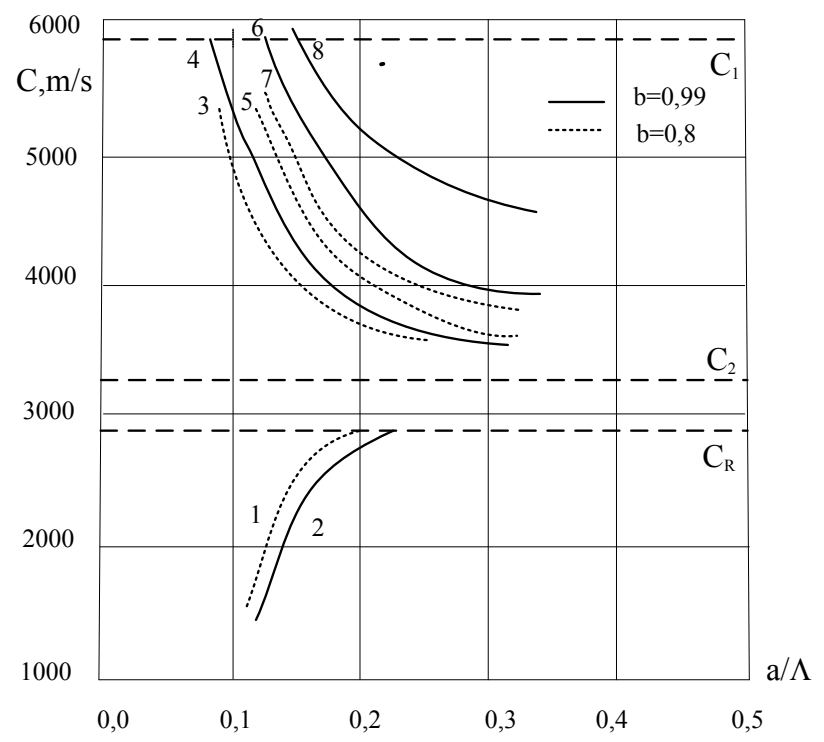

Figure 5. The phase velocities of three-dimensional flexural waves in steel cylindrical shells

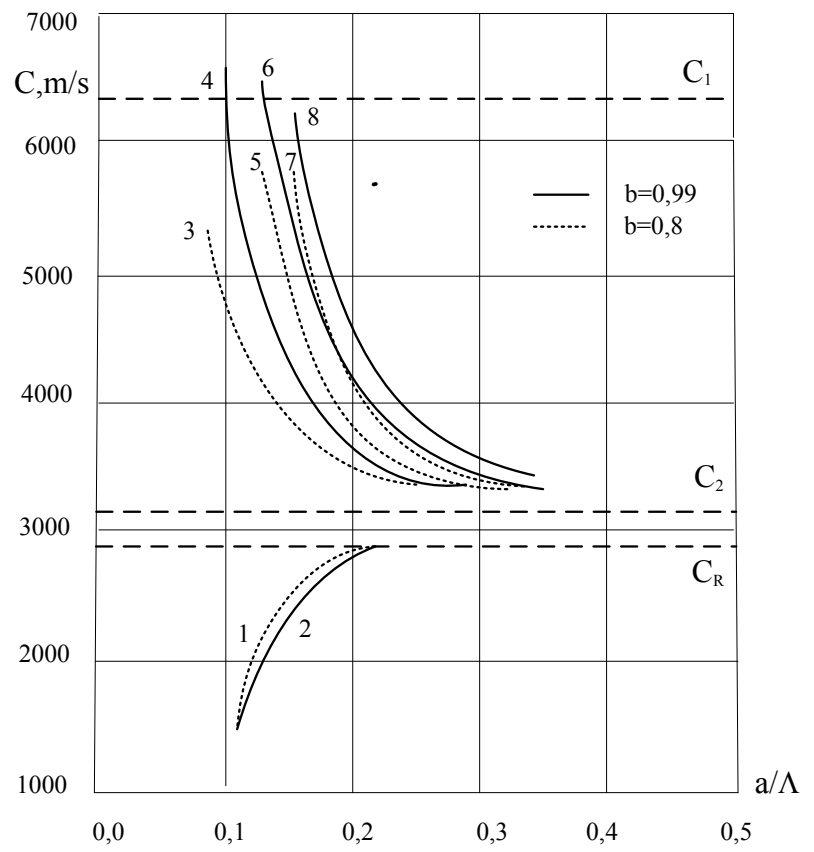

Figure 6. The phase velocities of three- dimensional flexural waves in aluminium cylindrical shells 


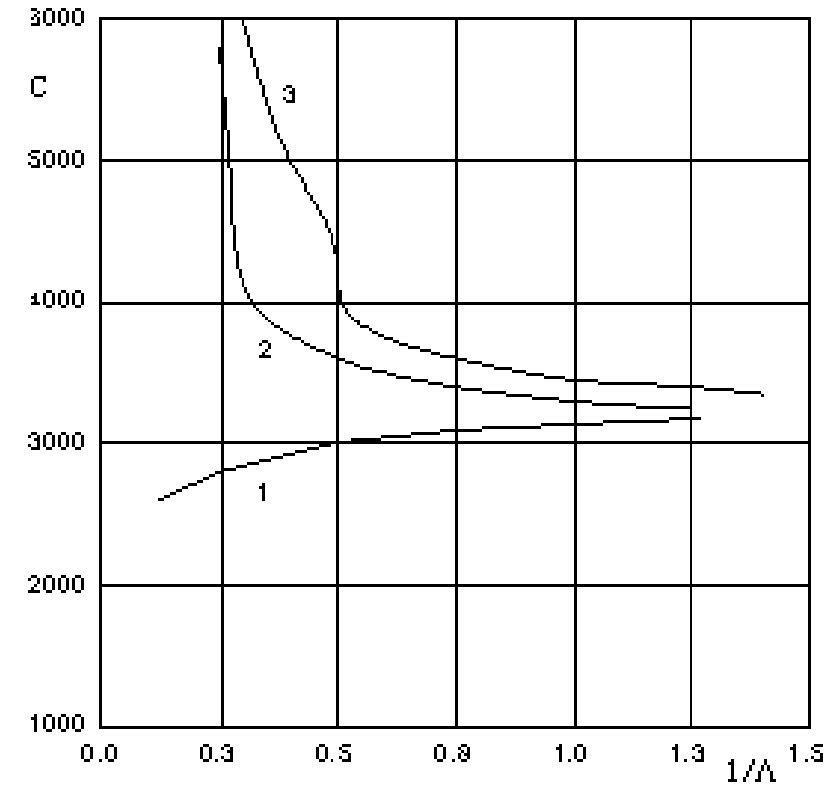

Figure 7. The phase velocities of first three forms of flexural waves in the steel cylindrical bar

\section{Conclusions}

The decision of a three-d imensional task of radiation of a sound by a cylindrical and spheroidal shells, which is raised by turbulent pulsations of a liquid flow is received. The characteristics of radiation by a spheroidal shell under action of such sources are calculated.

The characteristic equations for wave numbers of the three-dimensional axial flexural waves, raised in an cylindrical shell and bar are received. The dispersive curves of phase velocities of the various forms of these waves for steel and aluminium shells of various thickness and for steel cylindrical bar are submitted.

\section{REFERENCES}

[1] I. Ja. Miniovich, A.D.Pernik, V.S. Petrovsky. Hydrodynamical sources of sound. L.: Shipbuilding, 1972.P.480.

[2] S.L. Ilmenkov, A.A. Kleshchev. Radiation elastic bodies spheroidal form and bond his with diffraction of sound. // Higher school collection. Ships acoustics. 1989. P.15-21. Leningrad shipbuilding institute.

[3] S.L. Ilmenkov, A.A. Kleshchev, I.I. Klukin, S.B. Rumjantsev. Radiation of elastic spheroid under act of point source. // Higher school collection. Ships systems.1989.P.100-104. Leningrad shipbuilding institute.

[4] A.A. Kleshchev. Hydroacoustic scatterers. // The second publication. Saint-Petersburg. Prima, 2012, P.267. Scientific\&Academic Publishing (SAP).

[5] A.A. Kleshchev. Diffraction and propagation of waves in elastic mediums and bodies. S-Pb.: Vlas. 2002. P.156.

[6] L.M. Ljamshev. To question about principle of reciprocity.
P.Ac.Sc. USSR. 1959.T.125. P.1231-1234.

[7] A.A. Kleshchev. Diffraction of sound radiated by a point source on an elastic cylindri cal shell. // J. of Techn. Acoust. 1997.V.3.N4.P.20-23.

[8] E.L. Shenderov. Wave problems of hydroacoustic. L.: Shipbuilding, 1972.P.350.

[9] L.M. Brehovskikh. Waves in flaky mediums. M.: Publ. House Ac.Sc. USSR, 1957. P.502.

[10] L. Felsen, U.N. Markuvits. Radiation and scattering of waves. M.: World, 1978,V.1-2.

[11] A.A. Kleshchev. Diffraction of sound by a point source on an elastic cylindrical shell. // Acoust. Journ. 2004. V.50. № 1. P.86-89.

[12] Debye P. Das Verhalten von Lichtwellen in der Nähe eines Brennpunktes oder einer Brennlinie. //Ann. Physik. 1909.V.30.N4.S.755-776.

[13] V.A. Fok. Problems of diffraction and propagation of electromagnetic waves. M.: Soviet radio, 1970. P.517.

[14] A.A. Kleshchev, I.I. Klukin. About bend waves in elastic circular cylindrical bar. // Higer school collection. 109. P. 3-5.

[15] A.A. Kleshchev. Against the phase velocities of flexural waves in cylindrical shell //J. of Techn. Acoust. 1997. V. 3. N4. P. $16-19$.

[16] A.A. Kleshchev. Diffraction of sound radiated by a point source on an elastic cylindrical shell // J. of Techn. Acoust. 1997. V. 3. N4. P. 20 - 23.

[17] Buchwald V. T. Rayleich waves in transversely isotropic media. // Quart. Journ. Mech. and Applied. Math. 1961. V. XIV. Pt. 3. P. 293 - 317.

[18] Ahmad F. Guided waves in a transversely isotropic cylinder immersed in a fluid. // J.A.S.A. 2001. V. 109. N3. P. 886 890.

[19] Fan Y., Sinclair A. N., Honarvar F. Scattering of a plane acoustic wave from a transversely isotropic cylinder encased in a solid elastic medium.//JASA.1999.V106.N3.Pt.1.P.1229 -1236 .

[20] A.A., Kleshchev, I.I. Klukin. About bend waves in elastic cylindrical bar. // Higer school collection. 1975.109. P. 3-5.

[21] A.A., Kleshchev, I.I. Klukin. Ships acoustics. L.: Shipbuilding, 1982. P.144.

[22] S.L. Ilmenkov, A.A. Kleshchev, Phase velocities of bend wave of nonzero forms of thin elastic circular infinite cylindrical bar. // J.of Techn. Acoust. 1999. v.4. № 1. P.14-17.

[23] S.L. Ilmenkov, A.A. Kleshchev, Phase velocities of bend wave of nonzero modes of elastic infinite cylindrical shell. // J.of Techn. Acoust. 1999. v.4. № 1. P.18-20.

[24] S.L. Ilmenkov, A.A. Kleshchev, Phase Velocities of Flexural, Longitudinal and Torsional waves in isotropic cylindrical shell (the correct solution) // Proceedings of the X Session of the Russian Acoustical Society. M.: GEOS. 2000, V.1, P 210-214.

[25] S.L. Ilmenkov, A.A. Kleshchev. Phase Velocities of Flexural waves in isotropic cylindrical shell of any thickness. // Proceedings of the X1 Session of the Russian Acoustical Society. M.: GEOS. 2001, V.1, P 239-241. 
[26] A.A. Kleshchev, K.A. Surgailo. Phase Velocities of elastic waves in isotropic cylindrical bar of any thickness. // Proceedings of the X1 Session of the Russian Acoustical Society. M.: GEOS. 2001, V.1, P. 236-238.

[27] S.L. Ilmenkov, A.A. Kleshchev, K.A. Surgailo. Phase Velocities of elastic waves in bars and shells // Proceedings of the Sc. Conf. GMTU, S-Pb. 2003 (is not issuded).

[28] A.A. Kleshchev. Debye's and "such as Debye's" potentials in the problems of diffraction, radiation and propagation of elastic waves // Proceedings of the V Int. Conf. «Morintech2003». 2003. V.2. S-Pb.: P.55-61.

[29] A.A. Kleshchev. About phase velocities of elastic waves in thin axissymmetrical isotropic cylindrical shell. // Proceedings of the X Session of the Acoustic society of Russia. 2000. V.1. M.: GEOS.P.206-210.

[30] A.A. Kleshchev. About phase velocities of elastic waves in thin ortotropic cylindrical shell. // Proceedings of the $\mathrm{X}$ Session of the Acoustic society of Russia.2001.V.1.M.: GEOS. P.241-244.

[31] A.A. Kleshchev. Dispersion equations of a displacement vectors of differents forms of elastic waves in isotropic and anizotropic cylindrical shells.// Proceedings of the X111 Session of the Acoustic society of Russia.2003.V.1.M.: GEOS..P. 255-257. 